\title{
Size effects on flow stress behavior during electrically-assisted micro-tension in a magnesium alloy AZ31
}

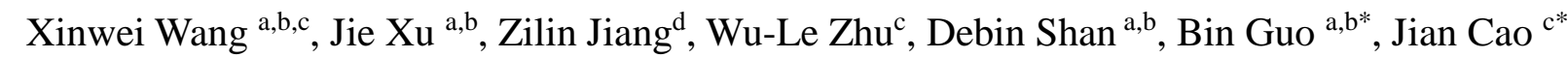

${ }^{a}$ Key Laboratory of Micro-Systems and Micro-Structures Manufacturing of Ministry of Education, Harbin Institute of Technology, Harbin 150080, China

${ }^{b}$ School of Materials Science and Engineering, Harbin Institute of Technology, Harbin 150001,

China

${ }^{c}$ Department of Mechanical Engineering, Northwestern University, 2145 Sheridan Rd, Evanston, IL 60208,USA

${ }^{\mathrm{d}}$ Department of Materials Science and Engineering, Northwestern University, 2145 Sheridan Rd, Evanston, IL 60208,USA

*Corresponding author, E-mail address: guobin@ @it.edu.cn (B. Guo); ${ }^{*}$ Corresponding author, E-mail address: jcao@northwestern.edu (J. Cao).

\begin{abstract}
As one of the promising micro-manufacturing technologies, micro-forming has economical and ecological advantages in terms of mass and near-net-shape production. However, size effects increasingly affect material performances with scaling down geometry and process parameters and consequently hinder applications of micro-forming. Electrically-assisted (EA) micro-forming may have the potential to minimize the size effects. In order to investigate the size effects in the EA micro-forming, uniaxial tension tests were conducted on miniaturized AZ31 tensile samples with varying grain sizes and geometry sizes at a constant DC current density. It was found that the normalized flow stress reduction, i.e., decreases of flow stresses / flow stresses at room temperature (RT), increased with the decrease of the grain size and with the increase of the geometry size at the
\end{abstract}


constant current density of $91.1 \mathrm{~A} / \mathrm{mm}^{2}$. Additionally, tests for the isolation of bulk Joule heating such as oven-heated tests and air-cooled EA tests were also conducted on the smallest samples with the same grain size regime examined in the EA tests to comparatively analyze the effect of grain size on the flow stress behavior during EA micro-tension. It was found that the amount of the Hall-Petch slope decreases in order of the RT, the oven-heated and the EA micro-tension tests. Both the Hall-Petch effects in the EA tests and the air-cooled EA tests are insignificant, showing nearly levelled Hall-Petch curves across the grain size regime examined. Based on these observations, a composite material model taking account of localized Joule heating at grain boundaries was developed to qualitatively interpret the Hall-Petch slope differences in the above four conditions. It was found that the mechanism behind the effects due to electric current may not be exclusive to bulk thermal softening.

Keywords: electrically-assisted, size effect, Hall-Petch effect, electroplastic effect, micro-tension, magnesium alloy

\section{Introduction}

The development of micro-manufacturing technology was driven by the growing demands for non-silicon micro-systems in automotive, medical, electronic \& aerospace industries. As one of the promising micro-manufacturing technologies, micro-forming is an economical and ecological technique since it has the advantages of mass production and net shape manufacturing. However, material flow behaviors during micro-forming are influenced by size effects, leading to deviations in expected material performances and consequently hindering applications of micro-forming. For example, Raulea et al. [1] studied the size effects of thin aluminum sheets using multiple forming processes including micro-tension, micro-bending and micro-blanking, and the results showed that the yield stress and the forming force decreased with a decreasing number of grains over the sheet 
thickness. Similarly, Krishnan et al. [2] conducted micro-extrusions of pins with a final diameter ranging from $1.33 \mathrm{~mm}$ down to $0.57 \mathrm{~mm}$ using brass, and an interesting result was found that $60 \%$ of the extruded pins showed a tendency to curve when using the smallest die (i.e., $0.76: 0.57 \mathrm{~mm}$ ) and the largest original grain size of $211 \mu \mathrm{m}$. Xu et al. [3] found that the ratio of blanking clearance to grain size was an important influence parameter during micro-blanking, and particularly at a 1:1 ratio the ultimate shearing strength reached a peak value. These findings have proved that the size effects result from the inhomogeneous deformation behavior of individual grains since only a small number of grains with stochastic distributions are involved in micro-forming processes. A summary of the size effects in metallic material deformation can be found in a review paper by Vollertsen et al. [4]. In order to minimize and control the size effects in micro-forming processes, Eichenhueller et al. [5] investigated micro-upsetting and micro-extrusion at elevated temperatures, and the results showed that the flow stress scatter decreased, moreover, hardness measurements verified that local deformation was improved at the micro-scale due to temperature. This is probably because the additional slip systems are activated and the annihilations of dislocations and grain boundaries speed up at elevated temperatures. However, as the geometry size of workpieces decreases, the ratio of surface to volume increases, leading to a severely poor surface finish and short tool life at elevated temperatures. Therefore, another alternative process may be needed with the capabilities of both the size effects controlling and the tool life improvement in micro-forming.

Electrical-assisted (EA) micro-forming, in which electric currents pass through miniaturized workpieces while undergoing plastic deformation, could be the alternative process since electric current has been found to induce various effects on mechanical properties of metals. For example, Jones et al. [6] applied continuous direct current (DC) during the upsetting of Mg AZ31B-O, and showed that the maximum reduction of the upsetting force was up to around $90 \%$ as well as the 
fracture displacement was about 5 times as that in baseline tests at a current density of $40 \mathrm{~A} / \mathrm{mm}^{2}$. Apart from applying continuous DC current, pulsed current was more frequently used to eliminate the elastic springback during sheet bending processes. Sánchez et al. [7] studied the effects of electric current on the bending behavior of aluminum alloy and stainless steel using current pulses. The results showed that when applying the current pulses with $50 \mathrm{~A} / \mathrm{mm}^{2}$ density, $50 \mu$ s pulse duration and $200 \mathrm{~Hz}$ frequency, about $18 \%$ bending force reduction and about $21 \%$ springback force reduction were obtained in aluminum, and those for stainless steel were approximately $6 \%$ and $12 \%$, respectively.

The mechanisms behind the effects of electric current on material properties during EA forming are still controversial since it is difficult to experimentally demonstrate an athermal current-induced effect, i.e., electroplastic effect. As a matter of fact, this athermal effect has been reported firstly in a review paper by Troitskii and coworkers [8] who conducted a series of studies on a number of metal single and polycrystals using high densities of pulsed electricity. They suggested that an electric current could aid in plastic deformation due to an interaction between electrons and dislocations. The basic principle is that electrons can transfer energies to static defects as well as mobile defects by scattering off the obstacles when flowing through the lattice of metals, and the additions of energies in the vicinity of the defects may cause a series of effects at the microstructural level in terms of localized lattice expansion, increase of localized diffusion rate, localized heating and alteration of localized stress-strain field, etc. Conrad et al. [9] provided comprehensive background knowledge on the electroplastic effect in metals, and also studied the mechanisms using EA tension. It was found that the electroplastic effect could be due to two contributions: the increased elastic and plastic strain rates. The former resulted from the thermal expansion due to Joule heating, while the latter was caused by the enhancement of dislocation 
mobility through the action of drift electrons including electron wind, increase of the vibration frequency of dislocation segments, decrease of the stack fault energy and the activation energy of metals, etc. Afterwards, both Perkins et al. [10] and Andrawes et al. [11] isolated the Joule heating effect using traditional thermal tests and claimed that heating could not be the exclusive current-induced effect. The former showed that the EA compression stress of titanium at peak temperature was roughly half that of oven-heated specimens at a temperature of $20{ }^{\circ} \mathrm{C}$ above the peak temperature. The latter found that the thermal tension tests followed the same basic trend as the baseline tests with no obvious stress drops, while the EA tests caused a significant softening of $\mathrm{AlMg} 1 \mathrm{SiCu}$. More recently, Kim et al. [12] concluded that Joule heating effect on the softening of aluminum alloy under pulsed EA tension was not dominant and the recovery of dislocations was observed at a certain pulsed current density through microstructure observations using EBSD and XRD. Roh et al. [13] also experimentally studied the tensile electroplasticity of an aluminum alloy under a pulsed electric current, and it was given that the formability of the material was greatly improved with insignificant thermal effects.

All the above researchers stated that heating was not the exclusive influence of electricity on EA deformation behavior. However, some recent researches showed that Joule heating dominated the EA plastic flow and also indicated that the electroplastic effect may not exist. Magargee et al. [14] used air flow during the EA deformation to control the Joule heating temperature in a miniaturized specimen by enhanced convection. They did not observe the electroplasticity through a comparison of the flow behaviors of pure titanium between EA tensile tests with and without air cooling. Based solely on the Joule heating effect, they developed modified thermal-mechanical models, i.e., the Hollomon model and the Johnson-Cook model, to successfully predict the uniaxial true stress-strain response of pure titanium at various current densities. Jordan and Kinsey [15] 
investigated EA micro-bending of brass sheets and found that the electricity can reduce the strain gradient through the thickness of bending specimens, particularly for coarse grain structures. Moreover, they also did not observe the electroplastic effect when applying $40 \mathrm{~A} / \mathrm{mm}^{2}$ current density to three-point bending samples. Kinsey et al [16] did high speed EA tension of $1 \mathrm{~mm}$ thick 304SS and Ti-6Al-4V samples using a Kolsky bar, and no electroplastic effects were observed in the results for current densities up to $180 \mathrm{~A} / \mathrm{mm}^{2}$ and the strain rate of $10^{3} \mathrm{~s}^{-1}$ since at the high strain rate further increase of the dislocation speed caused by additional electrical energy would not be possible due to the viscous drag of dislocations.

It is worthwhile to note that most of the above mentioned studies were only based on the classic thermal effect (i.e., bulk Joule heating) and the classic athermal effect (i.e., electron wind) to account for the flow behavior during EA forming without considering the influence of grain size on EA stress-strain responses. As a matter of fact, some papers have shown that the grain size was an important influence factor that should be taken into account since it may cause micro-scale Joule heating (i.e., localized resistive heating) or reveal the electroplastic effect. For example, local intergranular cavitation and local grain boundary melting were found by Fan et al. [17] during the microstructure observation on EA tension samples of $70 / 30$ brass. It was also observed that decreasing grain size increased stress drops under a continuous DC current. Siopis et al. [18] conducted EA compression of specimens of $1 \mathrm{~mm}$ in diameter with varying grain sizes, and showed that the flow stress reduction caused by the electric current increased with the increasing of current density and the decreasing of grain size. Another work reported by Siopis and Kinsey [19] gave that the data scattering during micro-compression was reduced by the current, especially for the specimens with larger grain size.

We aim to investigate the size effect on the flow stress behavior during EA micro-tension of 
AZ31 magnesium alloy in this study, since previous researches mainly focused on conventional EA forming processes and less work was associated with the effects of grain size and geometry size on EA mechanical properties. Meanwhile, the mechanism of the size effect during EA micro-tension is another goal in this study for further understanding of current-induced effects. To accomplish this, the EA micro-tension tests of AZ31 specimens were conducted for four different grain sizes as well as four different geometry sizes at a constant current density. Additionally, experiments for the isolation of Joule heating including oven-heated tests and air-cooled EA tests were also conducted for the grain sizes same as those in the EA tests. Then, a comparative analysis of the size effects occurred during RT tests, EA tests, oven-heated tests and air-cooled EA tests makes the understanding of the current-induced effects possible. Particularly, the Hall-Petch effects for the RT, EA, oven-heated, and air-cooled tests were compared and analyzed. A composite material model was also established to account for the differences in the observed Hall-Petch slopes.

\section{Experimental Setup}

\subsection{Sample preparation}

Uniaxial micro-tension tests were performed on AZ31 magnesium alloy specimens which were prepared at Harbin Institute of Technology. The gauge length and gauge width of the dogbone samples are $2.5 \pm 0.01 \mathrm{~mm}$ and $1 \pm 0.01 \mathrm{~mm}$, respectively. In order to investigate the geometry size effect during EA micro-tension, samples with different geometry sizes, i.e., $1.5 \mathrm{~mm}, 2 \mathrm{~mm}, 3 \mathrm{~mm}$ in gauge widths and $3.75 \mathrm{~mm}, 5 \mathrm{~mm}, 7.5 \mathrm{~mm}$ in corresponding gauge lengths, were prepared. These tension specimens were cut from the axial cross-section of a $9.8 \mathrm{~mm}$ diameter AZ31 bar along the axial direction using electrical discharge machining. In order to avoid the effect of surface burning and oxidation during the sample cutting, the initial cutting thickness is $\sim 0.3 \mathrm{~mm}$, and then the samples were mechanically polished to a thickness of $\sim 0.2 \pm 0.05 \mathrm{~mm}$ for micro-tension tests. Prior 
to cutting, annealing was used to intentionally vary the grain size of the material. The grain sizes obtained by annealing at the temperatures of $400{ }^{\circ} \mathrm{C}, 500{ }^{\circ} \mathrm{C}, 550{ }^{\circ} \mathrm{C}$ and $600{ }^{\circ} \mathrm{C}$ for $2 \mathrm{~h}$ are listed in Table 1. Therefore, specimens with 1-3, 3-5, 7-13, 18-33 grains through the thickness, respectively, were obtained, which are considered as micro-scale specimens based on the classification given by Hansen [20].

Table 1. Heat treatment parameters and the corresponding grain sizes.

\begin{tabular}{ccccc}
\hline $\begin{array}{c}\text { Average grain size } \\
(\mu \mathrm{m})\end{array}$ & $\begin{array}{c}\text { Standard deviation } \\
(\mu \mathrm{m})\end{array}$ & $\begin{array}{c}\text { Approximate grains } \\
\text { through thickness }\end{array}$ & $\begin{array}{c}\text { Temperature } \\
\left({ }^{\circ} \mathrm{C}\right)\end{array}$ & $\begin{array}{c}\text { Time } \\
(\mathrm{h})\end{array}$ \\
\hline 99.8 & 24.9 & $1-3$ & 600 & 2 \\
43.3 & 4.4 & $3-5$ & 550 & 2 \\
17.6 & 2.3 & $7-13$ & 500 & 2 \\
7.1 & 1.1 & $18-33$ & 400 & 2 \\
\hline \hline
\end{tabular}

\subsection{Setup of four different test methods}

A desktop mechanical testing system at Northwestern University was used to conduct RT and EA experiments, which includes a scanning electron microscope (SEM) loading stage, a power supply and a noncontact measurement system. The SEM loading stage has a DC motor to drive two precisely aligned lead screws, which pulls specimens under a load cell of $100 \mathrm{~N}$ capacity with a resolution of $0.01 \mathrm{~N}$ for the measurement of deformation forces. A rectifier-based DC power supply with $3600 \mathrm{~W}$ peak-power was used to supply a preset current output during EA experiments. The positive and negative electrical leads of the power supply were securely attached to the upper and lower tension grips, respectively, passing electricity through the specimen from the upper grip to the lower grip. Ceramic washer and Telflon-covered steel screws were used to electrically insulate the SEM loading stage and the sensors from the charged grips. Note that the noncontact digital image correlation (DIC) method was used to compute strain since conventional strain measurement methods are not appropriate and accurate for miniaturized samples under EA deformation. A high 
resolution $\mathrm{CCD}$ camera, located on the front side of the loading stage, was used to capture the deformed images of patterned specimens which were then processed by a DIC software. Additionally, an infrared camera with a thermal resolution of $0.1{ }^{\circ} \mathrm{C}$ and temperature range of $-20 \sim$ $900{ }^{\circ} \mathrm{C}$, located on the back side of the SEM loading stage, was used to directly measure the temporal and spatial temperature on the surface of specimens during experiments. In order to avoid inaccurate measurements of the temperature due to the variable emissivity of shiny metallic surfaces, a high-temperature pure black paint (Zynolyte Hi-Temp Paint, Aervoe Industries Inc.) was sprayed on the back side of the specimens, forming a thin layer of coating with an uniform emissivity, and the infrared temperature measurements were initially calibrated using a thermocouple for comparison. This setup has been demonstrated to be successful for characterization of flow stress for pure titanium under EA uniaxial tension [14].

In order to make a direct comparison of thermal effects and other possible non-thermal effects due to electric current, both oven-heated tests and air-cooled EA tests were conducted in this study. The specimens in these tests had the same geometry size and grain size as those used in the EA tensile tests. For the setup of the oven-heated tensile tests, a MTS Sintech 20/G universal material testing machine with an integrated convection heating oven was used. The load cell used for deformation force measurements has $1000 \mathrm{lbs}$ capacity with a resolution of $0.1 \mathrm{~N}$. The preset temperature for the oven-heated tests was approximately the maximum temperature measured in the EA tests. Prior to the oven-heated tests, the oven was held at the preset temperature for $15 \mathrm{~min}$. Then specimens were mounted inside the oven and a total period of $15 \mathrm{~min}$ was given to heat the specimens and stabilize the specimen temperature. Afterwards, the oven-heated tests were conducted.

For the setup of the air-cooled EA tensile tests, we used the same system as that of the EA 
micro-tension tests. In addition to this, an air-cooled system was integrated to blow out a stream of air across the surface of the specimens while passing through electricity under tension. The air-cooled system had a compressed air supply with a pressure of $50 \mathrm{psi}$, a solenoid valve (Omega FSV 15 series) for controlling the amount of airflow and a flat-profile copper nozzle to uniformly cool the gauge area of the specimens. It has been used to effectively reduce Joule heating temperatures of pure titanium sheets under EA tension [14].

In this study, all the tests were conducted under a fixed strain rate of $0.01 \mathrm{~s}^{-1}$ until fracture. A continuous and constant-amplitude DC current was used to assist plastic deformation while a continuous uniaxial tension took place in the samples. As a result, the transient current density in the specimens increases with the decrease of cross-section area during tensile deformation. In order to investigate the grain size effect and the geometry size effect under the application of electricity, we used a constant initial current density (i.e., supplied current / initial cross-section area) of 91.1 $\mathrm{A} / \mathrm{mm}^{2}$ for all the EA micro-tension tests. Since the initial sharp increase of temperature due to Joule heating may have additional effects on the mechanical behavior of material, we started tensile deformations after the thermal equilibrium of the Joule heating (i.e., 5 - 10 s). It should be mentioned that all the tests presented in this paper were run at least 3 times for repeatability, and the strain measurements for all the tests were accomplished by DIC. The DIC software used for 2D strain field computation is Ncorr [21] which has been demonstrated to be an alternative to commercial 2D DIC packages [22].

\section{Results}

\subsection{EA micro-tension tests}

\subsubsection{Effect of grain size at constant current density}

Both the temporal and spatial distributions of the specimen surface temperature were measured 
using infrared camera in this study. Figure 1 shows a typical temperature spatial distribution in the gauge area of a $7.1 \mu \mathrm{m}$ grain size specimen at $5 \mathrm{~s}$ after the initiation of tension. It is observed that the temperature spatial distribution is not uniform which is consistent with the results reported in the paper [14]. The center of the specimen is the hottest during deformation due to the symmetric configuration of uniaxial tension, as shown in the enlarged picture of Fig. 1, the hottest area is approximately $1 \mathrm{~mm}$ in length and $0.5 \mathrm{~mm}$ in width. In this paper, we use the temperature in the vicinity of specimen center to characterize the amount of bulk Joule heating in the EA tensile tests.

The maximum temperature histories of the specimens with different grain sizes during the EA micro-tension tests are shown in Fig. 2. To avoid random errors, the mean and the standard deviations of the measured temperatures were computed for characterization of the thermal behavior during the EA micro-tension tests. As it can be seen, the maximum temperatures of the samples tend to increase with time. This is mostly because the cross-section area of the specimens decreased with tension deformation, causing an increasing current density passing through the specimens. It is noted that the increasing rate of the temperature is relatively small at the initial deformation stage (i.e., $1-2{ }^{\circ} \mathrm{C} / \mathrm{s}$ ), but at the final deformation stage the rate sharply increases with time due to the necking and cracking in the material. An unobvious grain size effect on the bulk Joule heating is observed during the EA micro-tension when maintaining a consistent initial current density of $91.1 \mathrm{~A} / \mathrm{mm}^{2}$. All the maximum temperatures fall within a narrow interval of $75-100{ }^{\circ} \mathrm{C}$. However, it should be noted that the smaller grain size case shows higher maximum temperatures throughout most of the duration of the tests. The slightly higher temperature at the $99.8 \mu \mathrm{m}$ grain size case compared to $7.1 \mu \mathrm{m}$ case is statistically insignificant. This may be due to a higher resistivity of fine grains than that of course grains [23].

In order to comparatively study the effect of grain size on flow stress behavior under EA 
micro-tension, both the RT tension tests and the EA tension tests were conducted for specimens with four different grain sizes. We use the mean stress-strain curves as the representative mechanical responses of the RT and EA micro-tensile tests, as shown in Fig. 3 and Fig. 4, respectively. The error bars marked on the mean stress-strain curves for all cases are less than \pm 25 $\mathrm{MPa}$, indicating that the results are repeatable. The curves for larger grain sizes are somewhat scattered perhaps because the grain sizes are comparable with the specimen thicknesses and the flow behavior of the larger grains is heterogeneous. The similar results were also obtained by Siopis and Kinsey [19]. As expected for the RT tests in Fig. 3, increasing the grain size resulted in lower flow stress. This could be explained by the well-known Hall-Petch effect which is based on the pile-ups on slip planes at grain boundaries during plastic deformation. It is observed that this effect is quite obvious at RT, e.g., at a true strain of 0.02 , the flow stress of $7.1 \mu \mathrm{m}$ grain specimens is approximately 1.5 times that of $99.8 \mu \mathrm{m}$ grain specimens.

As shown in Fig. 4, the flow stresses for different grain sizes all reduced compared with those in Fig. 3 when a current density of $91.1 \mathrm{~A} / \mathrm{mm}^{2}$ was applied in micro-tension. It is of interest to note that the effect of grain size on the flow stress in the EA tension tests is much smaller compared with that in the RT tension tests. For example, at true strain of 0.02 , the difference of the flow stresses between the $7.1 \mu \mathrm{m}$ case and the $99.8 \mu \mathrm{m}$ case is around $20.6 \mathrm{MPa}$, while it is $83.5 \mathrm{MPa}$ for the RT tension tests. This result indicates that the applied electric current may counteract the Hall-Petch effect. In order to make direct comparisons of the flow stresses, a normalized flow stress reduction $\Delta \sigma_{\text {norm }}$, i.e., decreases of flow stresses / flow stresses at RT, was used to characterize the differences. The variations in the normalized flow stress reduction for various grain sizes at different true strains of EA tension specimens are shown in Fig. 5. As it can be seen, there is a systematic decrease in the normalized flow stress reduction when increasing the grain size of the specimens in EA 
micro-tension tests, i.e., $\sim 27 \%$ for $7.1 \mu \mathrm{m}$ and $\sim 7 \%$ for $99.8 \mu \mathrm{m}$ at the strain of 0.02 . Another feature should be noted in Fig. 5 is that the normalized flow stress reduction does not change much as the strain increases for the same grain size. The reason is that the strain hardening rate also does not change much during EA deformation perhaps because limited static dislocations are present in the miniaturized specimens.

\subsubsection{Effect of geometry size at constant current density}

It is important to understand the geometry size effect on the deformation behavior of materials in microforming. Therefore, specimens with various geometry sizes were tested using uniaxial tensions to study the mechanical and thermal behavior of AZ31 magnesium alloy during the passage of $91.1 \mathrm{~A} / \mathrm{mm}^{2}$ current density. As shown in Fig. 6 and Fig. 7, uniaxial tension behaviors of $43.3 \mu \mathrm{m}$ grain size samples with different geometry sizes are obtained without and with the application of electric current, respectively. It is observed in the case without current that the smaller geometry size causes the lower flow stress, which is consistent with the surface layer model [24] usually used to account for the size effect in microforming. The smallest case does not follow the trend probably because the microstructure starts to significantly affect the flow behavior.

An obvious inversed geometry size effect on the flow stress can be seen in the case of applying the constant current density of $91.1 \mathrm{~A} / \mathrm{mm}^{2}$. This effect is shown in Fig. 8 that the normalized flow stress reductions at different strains increase with the increase of the specimen gauge area, viz., $\sim 12.9 \%$ and $\sim 85.8 \%$ for the smallest and the largest samples, respectively. Additionally, the variation of the maximum temperature with the specimen gauge area is also shown in Fig. 8. It can be seen that the temperature increases with increasing the specimen geometry size at the same current density. One reason for this is that the heat loss rate is higher for smaller workpieces since the surface-to-volume ratio increases and the heat conduction distance decreases with the 
miniaturization. Besides, it should be mentioned that the current-induced heating is probably different from the conventional bulk heating since Joule heating effects are dependent on material resistivity mainly caused by the scattering of electrons off defects in the lattice such as dislocations, grain boundaries and voids, etc. These effects should be more significant in larger specimens because larger amounts of defects are included in the deformed area, causing more localized atomic-level resistive heating [25] in the vicinity of the disordered lattice. It should be noted that both the maximum temperature and the normalized flow stress reduction approximately have linear relationships with the gauge area, which also shows a very strong correlation with each other.

\subsection{Isolation of Joule heating}

It is difficult to infer whether the EA flow behavior is caused by the thermal Joule heating effect, the non-thermal electroplastic effect or both. For a comparative study of the effects, both oven-heated micro-tension tests and air-cooled EA micro-tension tests were conducted. The basic difference is that minutes ( $15 \mathrm{~min}$ in our study) are needed to reach a desired temperature in the former tests, while the latter ones only take a few seconds (5 - $10 \mathrm{~s}$ in our study). Thus, it helps to isolate possible current-induced effects. The smallest geometry size was used in both cases to avoid the geometry size effect under the application of electric current. Then, a direct comparison between the normalized flow stress reductions in both cases at various grain sizes makes the understanding of the current-induced effects possible.

\subsubsection{Oven-heated micro-tension tests}

The temperature of the oven-heated tests was set as $100{ }^{\circ} \mathrm{C}$ which is slightly higher than the maximum Joule heating temperatures generated during all the EA tension tests in this study (as shown in Fig. 2). The temperature was reached and maintained by a convection fan inside the oven and monitored by a thermocouple placed beside samples. This over-estimation of the thermal Joule 
heating effect helps to indirectly study the non-thermal effect due to electric current. As shown in Fig. 9, the flow stresses for all cases in the oven-heated micro-tension tests are closer to the results in the RT tests (Fig. 3) than those in the EA micro-tension tests (Fig. 4).

As shown in Fig. 10, the normalized flow stress reductions for each grain size at different strains are less than those in Fig. 5. For example, at the strain of 0.02, $\Delta \sigma_{\text {norm }}$ in the oven-heated tests of the $7.1 \mu \mathrm{m}$ grain size specimens is around $10.3 \%$, whereas $27.4 \%$ is obtained in the EA tests. As shown in Fig. 11, the normalized flow stress reductions at the strain of 0.02 and 0.03 for the four different grain sizes are compared among the EA tests, the oven-heated tests and the air-cooled tests. It should be noted that the normalized stress drops during the oven-heated tests vary little with the grain size except for the $99.8 \mu \mathrm{m}$ grain size, most of which fall in the vicinity of $\sim 10 \%$. This uniform softening behavior regardless of the grain size implies that conventional heating tends to be uniform in bulk materials without micro-scale thermal gradient. Compared with the results in the air-cooled EA micro-tension tests, it further verifies the assumption of the localized Joule heating during the passage of electric current. The anomalous behavior for the largest grain size samples is probably due to the stochastic properties of multi-grains through the thickness.

\subsubsection{Air-cooled EA micro-tension tests}

During the air-cooled EA micro-tension tests, the initial current density was $52 \mathrm{~A} / \mathrm{mm}^{2}$ at which the Joule heating effect was not so significant that the cooling system could effectively cool the specimens down to the RT. Additionally, the air-cooling started prior to the electric current supply to avoid heating the specimens far beyond the RT. The results for different grain sizes are shown in Fig. 12 under the air-cooled condition. It can be seen that the stress-strain curves overlap each other except for the $99.8 \mu \mathrm{m}$ grain size, and the maximum Joule heating temperatures after air cooling are nearly the same as the RT. As shown in Fig. 13, we can see that the smallest grain size 
causes the highest stress drop in the air-cooled EA tension. Since the air-cooling method only has the ability to cool down the temperature on the surface of specimens through enhanced heat convection, the temperature distributions inside the material may not be affected, especially in the vicinity of grain boundaries, i.e., the localized atomic-level Joule heating which provides additional energies to dislocation movements through obstacles. This assumption has been proved by Fan et al [17] that local intergranular cavitation and local grain boundary melting occurred during EA deformation based on microstructure observations. Therefore, the smallest grain size would have the largest stress drop. For the larger grain sizes such as $43.3 \mu \mathrm{m}$ and $99.8 \mu \mathrm{m}$, the stress drops are negative and tend to be scattered, which is probably due to the anisotropic properties of some individual grains. Meanwhile, it can be inferred that the nonthermal electroplastic effect (i.e., the electron wind) may not alter the material state much. Specifically in the case of the largest grain size, we could exclude or neglect the heating effect since the maximum bulk temperature was air cooled to the RT and the localized Joule heating could be limited due to few grain boundaries included in a small material volume. Also, the flow stress curve of the largest grain size specimens under the air-cooled EA tests would overlap with that in the RT tests. Thus, the possible reason for these is that the nonthermal electroplastic effect could be negligible.

\section{Discussion}

The Hall-Petch relationships in the RT, EA, oven-heated and air-cooled EA tests as well as their corresponding Hall-Petch slopes are presented and discussed in Section 4.1. A composite material model is proposed to qualitatively account for the differences of the Hall-Petch slopes in Section 4.2. Then, a comparison between the experimental and the calculated Hall-Petch slopes is presented in Section 4.3. 


\subsection{Hall - Petch effect}

The strong influence of grain size on the mechanical properties of materials has been continuously investigated since the early work of Hall [26] and Petch [27]. A fact of increasing yield strength with decreasing grain size has been verified for many metals and alloys, which was further developed with considering the strain hardening effect as,

$$
\sigma_{(\varepsilon, d)}=\sigma_{0(\varepsilon)}+\frac{k_{h p(\varepsilon)}}{\sqrt{d}}
$$

where $\sigma_{(\varepsilon, d)}$ is the yield stress for a given strain $\varepsilon$ and grain size $d . \sigma_{0(\varepsilon)}$ and $k_{h p(\varepsilon)}$ are the lattice friction stress and the Hall-Petch slope for the given strain, respectively. The Hall-Petch relationships under the EA, RT, oven-heated and air-cooled micro-tension tests are directly compared, as shown in Fig. 14. It can be seen that the relationships between the yield stress and the grain size for four types of the tests all follow Eq. (1). As expected, the conventional thermal activation in the oven-heated case causes weaker grain boundaries compared to the stronger grain boundary strengthening in the RT case, while the unexpected result is that the Hall-Petch line in the EA case nearly levels off across the grain size regime examined. The air-cooled EA case also shows the same Hall-Petch behavior as that of the EA case. It can be inferred that grain boundaries are the weakest under the EA condition.

In order to further study the differences of the Hall-Petch relationships, the Hall-Petch slopes at different offset strains in the EA, RT, oven-heated and air-cooled micro-tension tests were obtained from the linear regression of the experimental data, as listed in Table 2. It is observed that the Hall-Petch slopes increase with increasing plastic deformation, indicating a stronger grain size effect at higher strain level. For example, the Hall-Petch slope at the offset strain of 0.01 in the RT tension tests is $267 \mathrm{MPa} \mu \mathrm{m}^{0.5}$, while $312.8 \mathrm{MPa} \mu \mathrm{m}^{0.5}$ is obtained at 0.02 offset strain, which fall in 
the range reported by Jain et al. [28]. The increase of the Hall-Petch slope with strain is perhaps caused by increasing twinning with strain since Wang et al. [29] claimed that Hall-Petch slope for twinning was much larger than that for dislocation slip. The differences of the Hall-Petch slopes between the RT and oven-heated tests are relatively consistent throughout the offset strains, i.e., about $110 \mathrm{MPa} \mu \mathrm{m}^{0.5}$, which is probably caused by the uniform conventional heating and thermal activation of additional slip systems in materials. It is worth noting in Table 2 that the amount of the Hall-Petch slope decreases in order of RT, oven-heated and EA micro-tension tests.

An especially interesting result is that the amount of the Hall-Petch slope is $60-200 \mathrm{MPa}$ $\mu \mathrm{m}^{0.5}$ lower in the EA tension tests than that in the oven-heated tests. This observation indicates that the influence of electric current on mechanical behavior is not considered to be exclusive to bulk heating. Meanwhile, we should also exclude the effect of recrystallization in all cases since the bulk temperatures $\left(\leq 100^{\circ} \mathrm{C}\right)$ are far below the recrystallization temperature, i.e., $250-450{ }^{\circ} \mathrm{C}[30$ ], of AZ31 in hot working. Moreover, considering the slight differences between the EA tests and the air-cooled EA tests, i.e., less than $40 \mathrm{MPa} \mu \mathrm{m}^{0.5}$, we can infer that localized heating at grain boundaries probably plays an additional role in softening materials. Particularly, electrical flow may transfer energies to defects in the form of heat (i.e., localized heating) or kinetic energy (i.e., electron wind) by the scattering of electrons off the defects. These effects would be more significant near grain boundaries due to their more disordered atomic arrangements. It is reported that the energy provided to a dislocation core in magnesium due to localized heating is about seven orders of magnitude of that caused by electron wind [25]. Therefore, the most probable effect that could account for the difference between the EA case and the oven-heated case is localized Joule heating as it could weaken the strength of grain boundaries further through reducing pile-ups against grain boundaries or grain boundary melting [17]. 
Bata and Pereloma [31] proposed that the Hall-Petch slope was proportional to the difference of the strain energies between a dislocation in bulk material and that at a grain boundary. Based on this theory, we can conclude that the Hall-Petch slope should increase with strain because plastic deformation could accumulate energy difference between bulk material and grain boundary, which should be especially severe for magnesium alloys with the HCP crystal structure due to the preferential dislocation slip on the basal planes of neighboring grains. However, localized heating may relieve the difference in the strain energy, which further verifies the reason for the lowest Hall-Petch slopes in the EA tension tests.

Table 2. Hall-Petch slopes at various offset strains in the RT, EA, oven-heated and air-cooled EA tests.

\begin{tabular}{|c|c|c|c|c|c|c|c|c|}
\hline \multirow{2}{*}{$\begin{array}{l}\text { Offset } \\
\text { strain }\end{array}$} & \multicolumn{2}{|c|}{ RT test } & \multicolumn{2}{|c|}{ Oven-heated test } & \multicolumn{2}{|c|}{ EA test } & \multicolumn{2}{|c|}{ Air-cooled EA test } \\
\hline & $k_{h p}{ }^{\mathrm{a}}$ & $s_{k}^{\mathrm{b}}$ & $k_{h p}$ & $s_{k}$ & $k_{h p}$ & $s_{k}$ & $k_{h p}$ & $s_{k}$ \\
\hline 0.005 & 263.9 & 25.0 & 170.5 & 24.4 & 109.9 & 74.6 & 112.1 & 40.5 \\
\hline 0.01 & 267.0 & 44.7 & 166.2 & 22.3 & 59.6 & 41.7 & 68.7 & 63.6 \\
\hline 0.02 & 312.8 & 54.1 & 195.4 & 19.9 & 64.8 & 42.9 & 104.7 & 100.5 \\
\hline 0.03 & 348.6 & 58.3 & 229.4 & 15.7 & 77.0 & 46.3 & / & / \\
\hline 0.04 & 402.1 & 55.5 & 287.7 & 1.5 & 87.9 & 47.5 & I & I \\
\hline
\end{tabular}

${ }^{\mathrm{a}}$ The unit of the Hall-Petch slope is $\mathrm{MPa} \mu \mathrm{m}^{0.5}$.

${ }^{\mathrm{b}}$ It represents the standard deviation of the Hall-Petch slope.

\subsection{Modeling of Hall - Petch effect for EA micro-tension}

Generally, the process of yielding in materials can be divided into three stages, viz., (i) establishing localized stress concentration at grain boundaries due to the elastic anisotropy of adjacent grains; (ii) micro-yielding at the grain boundaries; (iii) macro-yielding throughout the materials. These assumptions are reasonable since some results on polycrystals [32] show that the hardening rate is higher in the regions close to the grain boundaries and triple points. In order to develop a relationship between the grain size and the macro-yield stress, Meyers et. al [33] considered the material as a composite consisting of a continuous network of grain-boundary 
material with flow stress $\sigma_{f g b}$ and discontinuous pockets of bulk material with flow stress $\sigma_{f b}$ based on the above assumptions. This composite model can be given as ,

$$
\sigma_{y}=A_{g} \sigma_{f g}+A_{g b} \sigma_{f g b}
$$

where $\sigma_{y}$ is the yield stress of polycrystalline materials, $A_{g}$ and $A_{g b}$ are the area fractions of the grain-boundary network and bulk pockets, respectively. It has been demonstrated to be an effective approach to model the grain size effect on material yield stress [34]. As shown in Fig. 15(a), the grains are assumed to be equiaxed hexagons with diameter $d$ and grain-boundary layer thickness $t$. Then, the area fractions of the grain-boundary network and bulk pockets can be determined by,

$$
\begin{gathered}
A_{g}=\left(1-\frac{2 t}{d}\right)^{2} \\
A_{g b}=1-A_{g}=\frac{4 t}{d}\left(1-\frac{t}{d}\right)
\end{gathered}
$$

Combining Eqs. (2) - (4), we have,

$$
\sigma_{y}=\sigma_{f g}+4\left(\sigma_{f g b}-\sigma_{f g}\right) \frac{t}{d}-4\left(\sigma_{f g b}-\sigma_{f g}\right) \frac{t^{2}}{d^{2}}
$$

Since different sections along the thickness direction of the specimens should produce different area fractions, it is more accurate if we replace $d$ and $t$ in Eq. (5) using their corresponding mean values $\bar{d}$ and $\bar{t}$ which have been given by Meyers and Ashworth [35] as,

$$
\frac{\bar{t}}{\bar{d}} \approx \frac{2 t}{d}
$$

Additionally, many researchers [33-35] have made a reasonable assumption of the relationship between $t$ and $d$, i.e.,

$$
t=\lambda \sqrt{d}
$$

since many materials follow the Hall-Petch relationship. $\lambda$ is a material constant. Based on Eqs. (5) - (7), we have, 


$$
\sigma_{y}=\sigma_{f g}+\frac{8 \lambda}{\sqrt{d}}\left(\sigma_{f g b}-\sigma_{f g}\right)-\frac{16 \lambda^{2}}{d}\left(\sigma_{f g b}-\sigma_{f g}\right)
$$

Equation (8) shows that the traditional Hall-Petch relationship is valid for larger grain sizes, while at smaller grain sizes the Hall-Petch curve would bend down until it levels off at $\sigma_{f g b}$. This phenomenon has been also reported in the thesis [36].

Generally, the grain boundary thickness is of the order of $0.5 \mathrm{~nm}$ [37], which is far less than the grain size $d$ in this study. Therefore, we can neglect $\frac{t^{2}}{d^{2}}$, i.e., $d^{-1}$ term in Eq. (8). Compared with Eq. (1), the Hall-Petch slope at RT can be given as,

$$
k_{h p}^{R T}=8 \lambda\left(\sigma_{f g b}-\sigma_{f g}\right)
$$

which is simply associated with the difference of the flow stresses between the grain boundary layer and the grain interior. If specimens are heated to a temperature $T$, we introduce a thermal softening factor $f_{T}\left(f_{T}<1\right)$ to characterize the decrease of the flow stress, i.e.,

$$
f_{T}=\frac{\sigma_{T}}{\sigma_{R}}
$$

where $\sigma_{R}$ and $\sigma_{T}$ are the flow stresses in the RT and oven-heated cases, respectively. Since the thermal softening factor at grain boundaries is consistent with that in grain interiors due to uniform heating throughout materials in the oven-heated case, the Hall-Petch slope can be given by,

$$
k_{h p}^{O v e n}=8 \lambda f_{T}\left(\sigma_{f g b}-\sigma_{f g}\right)
$$

For the EA case, as shown in Fig. 15(b), the grain boundaries (denoted as red color) would be hotter than the grain interiors (denoted as blue color) due to higher electrical resistivity at the grain boundaries [38]. Therefore, the Hall-Petch slope during EA tension cannot be expressed using an uniform thermal softening factor since localized Joule heating should be taken into account in addition to bulk heating. Magargee et al. [39] have established a relationship between the thermal 
softening factor and the effective current density during the EA tension of various metals and alloys, i.e.,

$$
f_{J}=\alpha \exp \left(-\beta \rho_{e} J^{2}\right)
$$

where $\rho_{e}$ represents the electrical resistivity, $\alpha$ and $\beta$ are material-dependent constants. It is found in Eq. (12) that the thermal softening factor in the EA case decreases with the increase of the current density and the electrical resistivity. In this study, the current density flowing through the grain boundaries is the same as that in the grain interiors. Since the electrical resistivity at the grain boundaries $\rho_{e}^{g b}$ is higher than that in the grain interiors $\rho_{e}^{g}$, we can have the relationship between the thermal softening factors of the grain boundaries $f_{J}^{g b}$ and the grain interiors $f_{J}^{g}$ as,

$$
f_{J}^{g b}=\alpha \exp \left(-\beta \rho_{e}^{g b} J^{2}\right)<\alpha \exp \left(-\beta \rho_{e}^{g} J^{2}\right)=f_{J}^{g}
$$

Taking account of the bulk Joule heating, we can approximately obtain the Hall-Petch slope during EA tension through applying different thermal softening factors, that is,

$$
k_{h p}^{E A}=8 \lambda\left(f_{J}^{g b} f_{T} \sigma_{f g b}-f_{J}^{g} f_{T} \sigma_{f g}\right)=8 \lambda f_{T}\left(f_{J}^{g b} \sigma_{f g b}-f_{J}^{g} \sigma_{f g}\right)
$$

where $f_{T}, f_{J}^{g b}$ and $f_{J}^{g}$ represent the contributions from the bulk Joule heating, the localized Joule heating at the grain boundaries and in the grain interiors, respectively. Since $f_{J}^{g b}<f_{J}^{g}<1$, we could qualitatively explain the lowest Hall-Petch slope in the EA tension tests.

\subsection{Comparison with experiments}

In order to predict the Hall-Petch slopes for various tests, at least three parameters have to be established, i.e., $\sigma_{f g b}, \sigma_{f g}$ and $\lambda$. We take the saturation stress of an ultrafine-grained (180 nm) magnesium alloy at RT as the $\sigma_{f g b}$ since the ultrafine-grained material is mainly composed of grain boundaries. The yield stress of $\mathrm{Mg}-\mathrm{Zn}$ single crystals at RT is used to represent $\sigma_{f g}$ due to no grain boundary strengthening. Table 3 lists the values of the three parameters used in the calculation. 
Table 3. Parameters used for the Hall-Petch slope model.

\begin{tabular}{lll}
\hline \hline Parameters & Value & Reference \\
\hline$\sigma_{f g}(\mathrm{MPa})$ & 415 & {$[40]$} \\
$\sigma_{f g}(\mathrm{MPa})$ & 80 & {$[41]$} \\
$\lambda\left(\mu \mathrm{m}^{0.5}\right)$ & 0.125 & {$[35]$} \\
\hline \hline
\end{tabular}

Note that the above method to calculate the Hall-Petch slope, i.e., Eq. (9), is independent of the strain level, and the calculated value is $335 \mathrm{MPa} \mu \mathrm{m}^{0.5}$. The second column of Table 2 shows the experimental values at RT at different strain levels. The averaged experimental value is $319 \mathrm{MPa}$ $\mu \mathrm{m}^{0.5}$, which is very closed to the calculated value. For the prediction of the Hall-Petch slope in oven-heated tests, it is necessary to determine the thermal softening factor $f_{T}$. Magargee et al. [14] found that $f_{T}$ was a linear function of the temperature by regression analysis of high-temperature flow stress data. Particularly, at the temperature in this study, the thermal softening factor is around 0.8. Based on Eq. (9) and Eq. (11), we can predict that the Hall-Petch slope in the oven-heated tests is 0.8 times that in the RT tests. However, as shown in Table 2, we have $k_{h p}^{O v e n}=(0.65 \sim 0.72) k_{h p}^{R T}$, which is lower than the predicted ratio. The over-estimation of Eq. (11) is perhaps because $\lambda$ may be also temperature dependent since dislocation pile-ups tend to recovery and annihilation at grain boundaries, causing a smaller grain-boundary layer thickness at elevated temperatures. Since it is difficult to experimentally measure the localized electrical resistivity at grain boundaries and in grain interiors, we assume $f_{J}^{g}=f_{T}=0.8$ and $\rho_{e}^{g b}=6 \rho_{e}^{g} \quad$ [25]. Then, we have,

$$
f_{J}^{g b}=\alpha \exp \left(-\beta \rho_{e}^{g b} J^{2}\right)=\alpha \exp \left(-6 \beta \rho_{e}^{g} J^{2}\right) \approx\left(f_{J}^{g}\right)^{6}
$$

since $\alpha$ is approximately equal to 1 [39]. Inserting these relationships and data into Eq. (14), the Hall-Petch slope in EA tension tests is predicted as $35.8 \mathrm{MPa \mu m}^{0.5}$ which has the same order of magnitude as the results listed in Table 2. The difference is probably due to the over-estimation of the difference in the electrical resistivity between grain boundaries and grain interiors. 


\section{Conclusions}

Miniaturized AZ31 magnesium alloy tensile specimens with various grain sizes and geometry sizes were subjected to continuous DC current while undergoing uniaxial tensile deformation. It was observed that at a constant current density the grain size effect on the bulk Joule heating was slight, while the geometry size significantly affected the Joule heating temperature. Meanwhile, the normalized flow stress reduction increased with the decrease of the grain size and the increase of the geometry size. In order to determine whether or not the bulk Joule heating effect can exclusively account for the above size effect occurred during the EA micro-tension, tests for the isolation of the bulk Joule heating such as the oven-heated tests and the air-cooled EA tests were run on the smallest samples with the same grain size regime examined in the EA tests. We found that the amount of the Hall-Petch slope decreased in order of RT, oven-heated and EA micro-tension tests. Moreover, the comparisons showed three interesting observations: (i) relatively consistent differences of the Hall-Petch slopes between the RT and oven-heated tests throughout the offset strains; (ii) higher amounts of the Hall-Petch slopes in the oven-heated tests than those in the EA tests; (ii) slight Hall-Petch effects for both the EA tests and the air-cooled EA tests. These observations indicate that bulk Joule heating is not the exclusive current-induced effect and there probably exists a difference between the traditional heating and the current-induced heating, i.e., localized Joule heating effect. Based on the assumption of localized Joule heating at grain boundaries, we extended the application of a composite material model for Hall-Petch relationship to qualitatively account for the Hall-Petch slope differences among the above four tests.

\section{Acknowledgements}

This work was supported by the National Natural Science Foundation of China under Grant No. 51475124, the China Scholarship Council under Grant No. 201306120136, and the National 
Science Foundation of the United States under Grants No. CMMI-1100787 and DMR-1121262. The authors are also grateful to Justin Blaber of the Georgia Institute of Technology for providing open source codes of Ncorr.

\section{References}

[1] L.V. Raulea, A.M. Goijaerts, L.E. Govaert, F.P.T. Baaijens, Journal of Materials Processing Technology, 115 (2001) 44-48.

[2] N. Krishnan, J. Cao, K. Dohda, Journal of Manufacturing Science and Engineering, 129 (2007) 669-676.

[3] J. Xu, B. Guo, C. Wang, D. Shan, International Journal of Machine Tools and Manufacture, 60 (2012) 27-34.

[4] F. Vollertsen, D. Biermann, H.N. Hansen, I. Jawahir, K. Kuzman, CIRP Annals-Manufacturing Technology, 58 (2009) 566-587.

[5] B. Eichenhueller, E. Egerer, U. Engel, The International Journal of Advanced Manufacturing Technology, 33 (2007) 119-124.

[6] J.J. Jones, L. Mears, J.T. Roth, Journal of Manufacturing Science and Engineering, 134 (2012) 034504.

[7] A.J.S. Egea, H.A.G. Rojas, D.J. Celentano, J.A. Travieso-Rodríguez, J.L. i Fuentes, Journal of Materials Processing Technology, 214 (2014) 2261-2267.

[8] N. Kir'yanchev, O. Troitskii, S. Klevtsur, Strength Mater, 15 (1983) 709-715.

[9] A. Sprecher, S. Mannan, H. Conrad, Acta Metallurgica, 34 (1986) 1145-1162.

[10] T.A. Perkins, T.J. Kronenberger, J.T. Roth, Journal of Manufacturing Science and Engineering, 129 (2007) 84-94.

[11] J.S. Andrawes, T.J. Kronenberger, T.A. Perkins, J.T. Roth, R.L. Warley, Materials and 
Manufacturing Processes, 22 (2007) 91-101.

[12] M.-J. Kim, K. Lee, K.H. Oh, I.-S. Choi, H.-H. Yu, S.-T. Hong, H.N. Han, Scripta Materialia, 75 (2014) 58-61.

[13] J.-H. Roh, J.-J. Seo, S.-T. Hong, M.-J. Kim, H.N. Han, J.T. Roth, International Journal of Plasticity, 58 (2014) 84-99.

[14] J. Magargee, F. Morestin, J. Cao, Journal of Engineering Materials and Technology, 135 (2013) 041003.

[15] A. Jordan, B.L. Kinsey, Journal of Materials Processing Technology, 221 (2015) 1-12.

[16] B. Kinsey, G. Cullen, A. Jordan, S. Mates, CIRP Annals-Manufacturing Technology, 62 (2013) 279-282.

[17] R. Fan, J. Magargee, P. Hu, J. Cao, Materials Science and Engineering: A, 574 (2013) 218-225.

[18] M.S. Siopis, B.L. Kinsey, N. Kota, O.B. Ozdoganlar, Journal of Manufacturing Science and Engineering, 133 (2011) 064502.

[19] M.S. Siopis, B.L. Kinsey, Journal of Manufacturing Science and Engineering, 132 (2010) 021004.

[20] N. Hansen, Acta Metallurgica, 25 (1977) 863-869.

[21] J. Blaber, B. Adair, A. Antoniou, Experimental Mechanics, (2015) 1-18.

[22] H. Ramesh, M. Ramji， 9th International Symposium on Advanced Science and Technology in Experimental Mechanics, 2014.

[23] D. Joshi, K. Sen, Solar Cells, 9 (1983) 261-267.

[24] M. Geiger, M. Kleiner, R. Eckstein, N. Tiesler, U. Engel, CIRP Annals-Manufacturing Technology, 50 (2001) 445-462.

[25] W.A. Salandro, J.J. Jones, C. Bunget, L. Mears, J.T. Roth, The Effect of Electric Current on 
Metals, Electrically Assisted Forming, Springer, 2015, pp. 37-54.

[26] E. Hall, Proceedings of the Physical Society. Section B, 64 (1951) 747.

[27] N. Petch, J. Iron Steel Inst., 174 (1953) 25-28.

[28] A. Jain, O. Duygulu, D. Brown, C. Tomé, S. Agnew, Materials Science and Engineering: A, 486 (2008) 545-555.

[29] H.-Y. Wang, E.-S. Xue, W. Xiao, Z. Liu, J.-B. Li, Q.-C. Jiang, Materials Science and Engineering: A, 528 (2011) 8790-8794.

[30] J. Tan, M. Tan, Materials Science and Engineering: A, 339 (2003) 124-132.

[31] V. Bata, E.V. Pereloma, Acta Materialia, 52 (2004) 657-665.

[32] G.T. Gray III, S.R. Chen, K.S. Vecchio, Metallurgical and Materials Transactions A, 30 (1999) 1235-1247.

[33] H.-H. Fu, D.J. Benson, M.A. Meyers, Acta Materialia, 49 (2001) 2567-2582.

[34] W. Chan, M. Fu, Materials Science and Engineering: A, 528 (2011) 7674-7683.

[35] M.A. Meyersm, E. Ashworth, Philosophical Magazine A, 46 (1982) 737-759.

[36] B. Yang, Chemicals, pharmaceuticals, biotechnology and materials science, Saarland University, Saarbrucken, 2007, pp. 167.

[37] L. Priester, Grain boundaries: from theory to engineering, Springer Science \& Business Media, 2012.

[38] T. Kino, T. Endo, S. Kawata, Journal of the Physical Society of Japan, 36 (1974) 698-705.

[39] J. Magargee, R. Fan, J. Cao, Journal of Manufacturing Science and Engineering, 135 (2013) 061022.

[40] F. Wen-bin, F. Wa, S. Hong-fei, Powder Technology, 212 (2011) 161-165.

[41] S. Ando, N. Harada, M. Tsushida, H. Kitahara, H. Tonda, Key Eng. Mater., Trans Tech Publ, 
2007, pp. 101-104. 


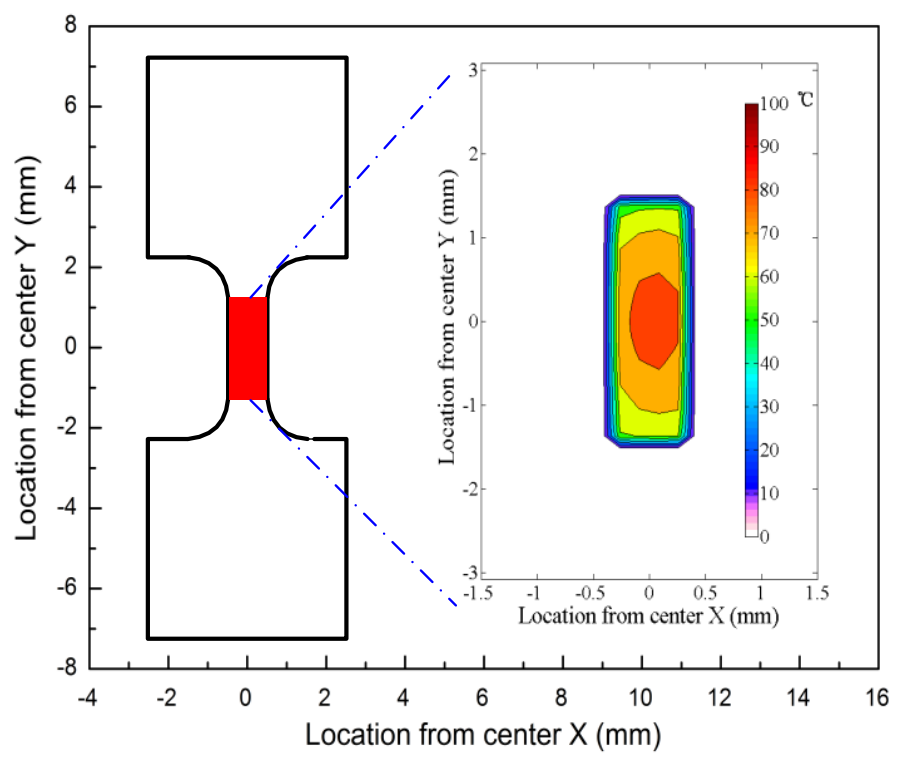

Fig. 1. A typical temperature spatial distribution of a EA micro-tension specimen (grain size: $7.1 \mu \mathrm{m}$, current density: $91.1 \mathrm{~A} / \mathrm{mm}^{2}$ ) at $5 \mathrm{~s}$ after the initiation of displacement. 


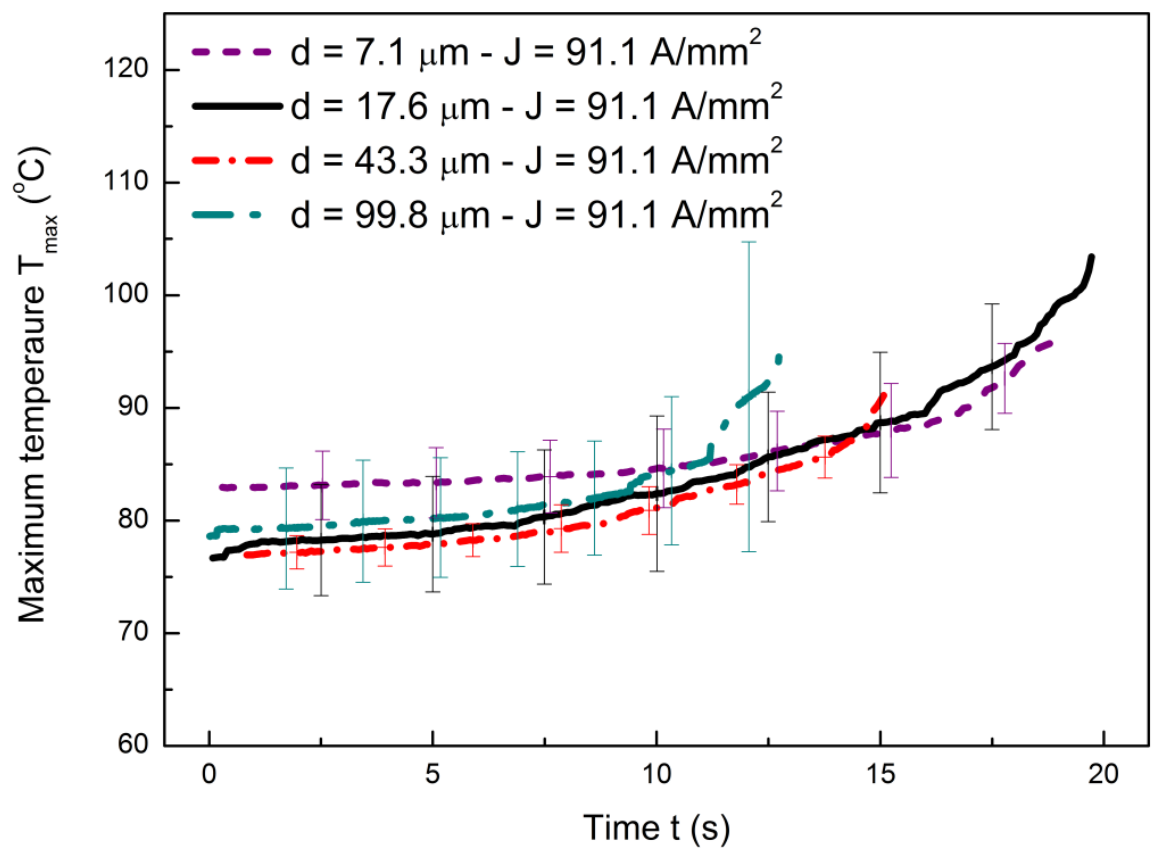

Fig. 2. Plots of maximum temperature against time for varying grain sizes during EA micro-tensile tests of the smallest samples at current density of $91.1 \mathrm{~A} / \mathrm{mm}^{2}$.

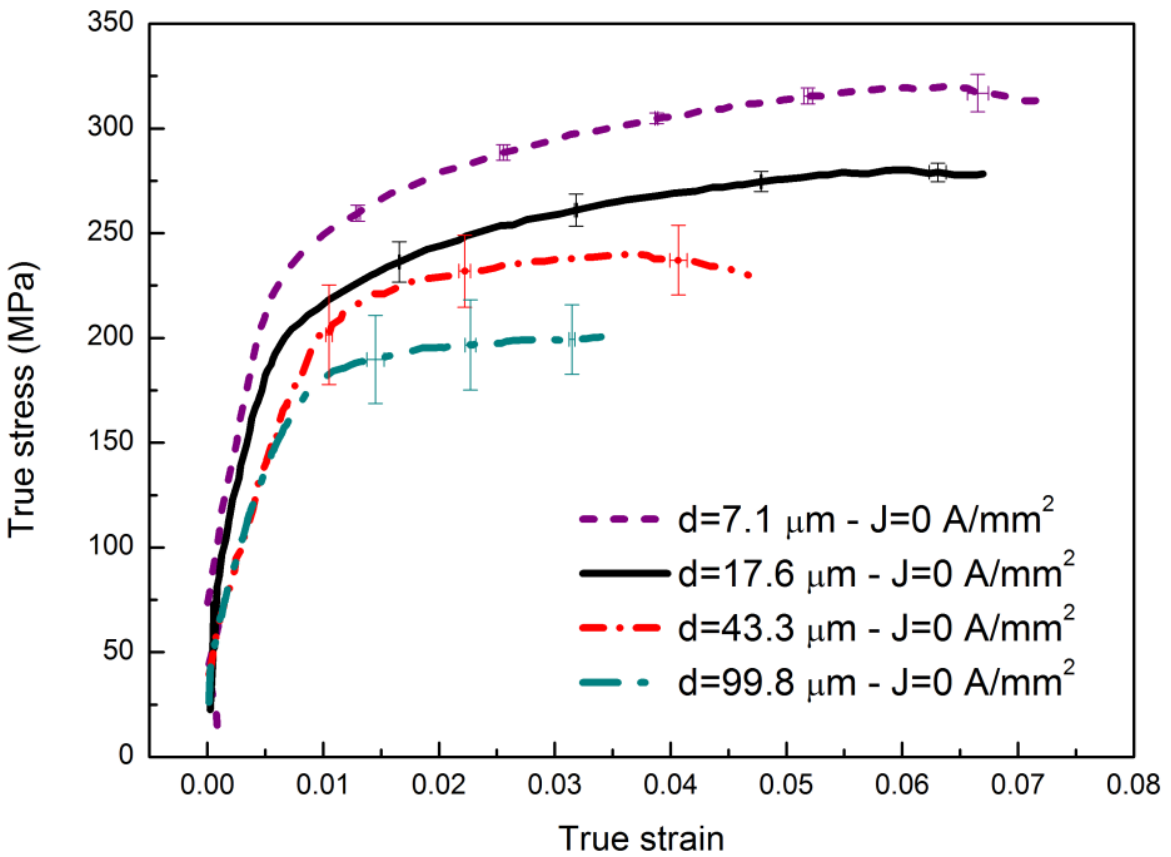


Fig. 3. True stress-strain curves for uniaxial micro-tension at RT of the smallest AZ31 specimens with varying grain sizes.

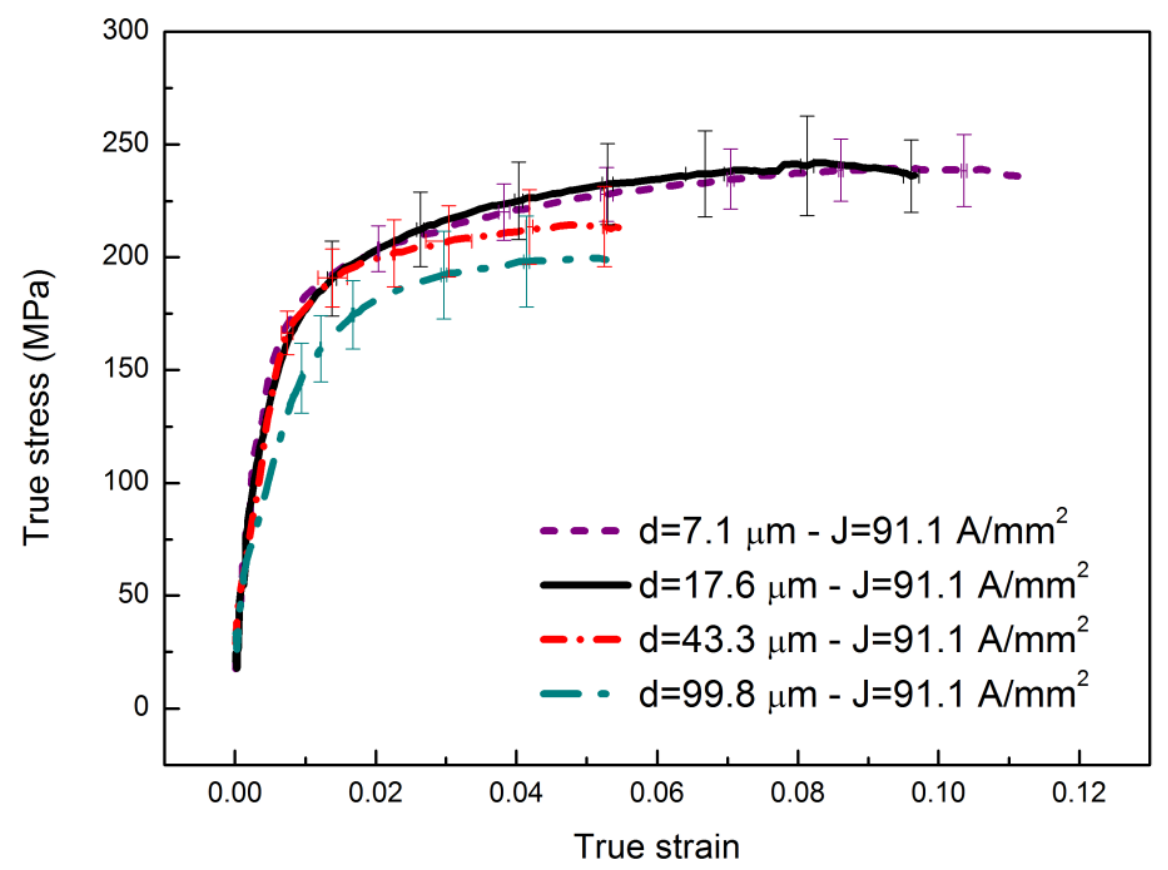

Fig. 4. True stress-strain curves during EA micro-tension of the smallest AZ31 specimens with varying grain sizes. 


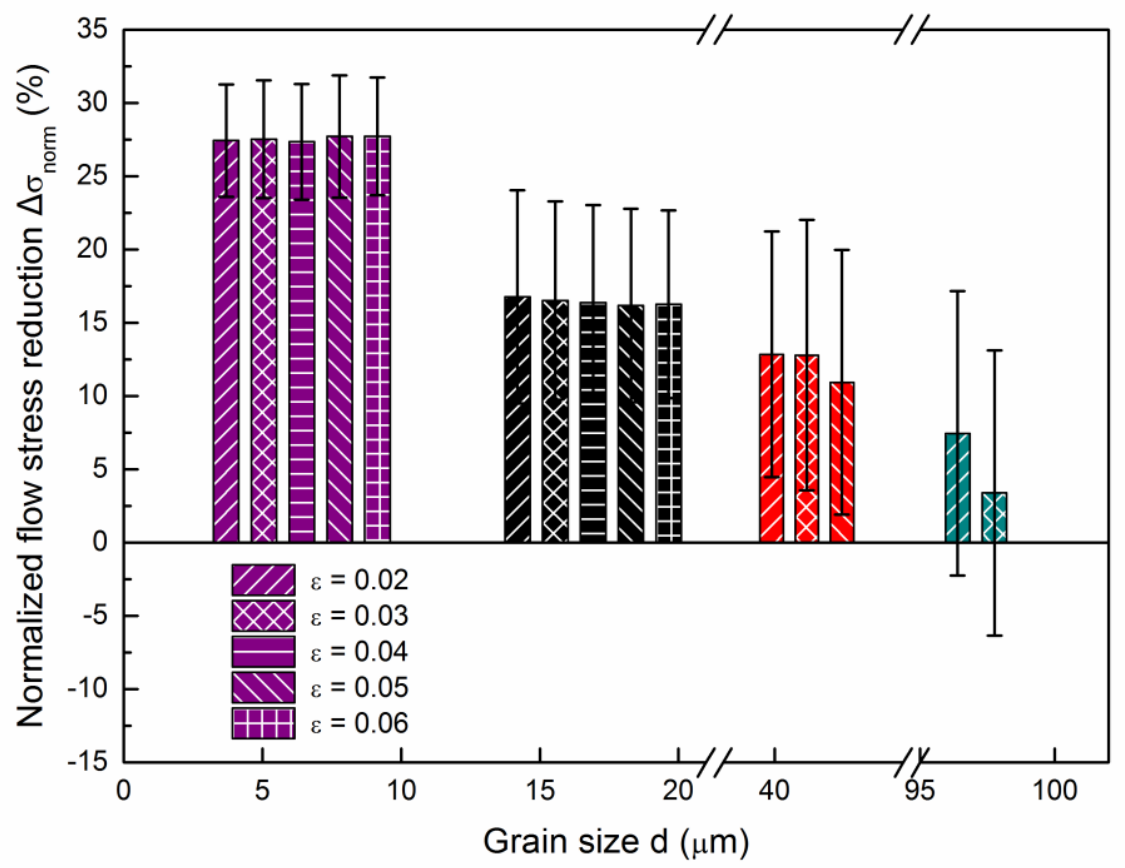

Fig. 5. Normalized flow stress reductions as a function of grain size at different strains for EA micro-tension. 


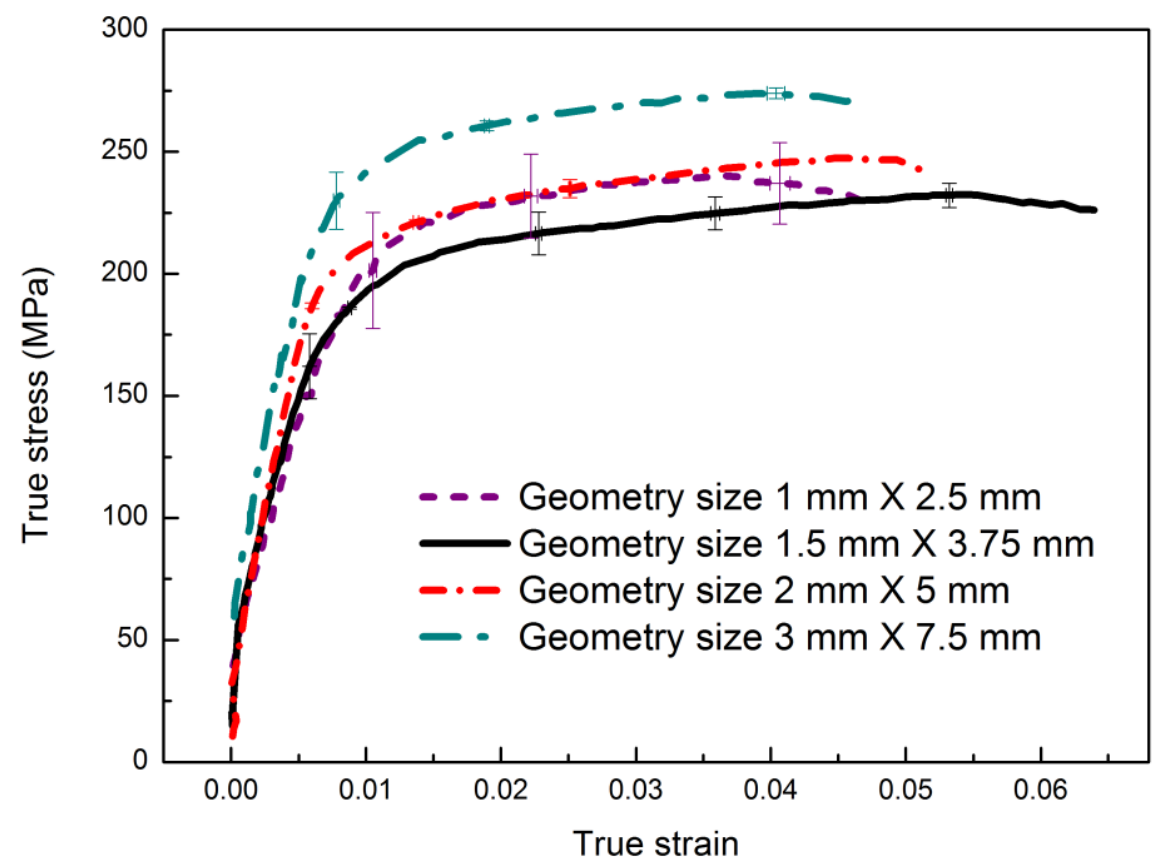

Fig. 6. True stress-strain curves for RT micro-tension tests of samples with various geometry sizes.

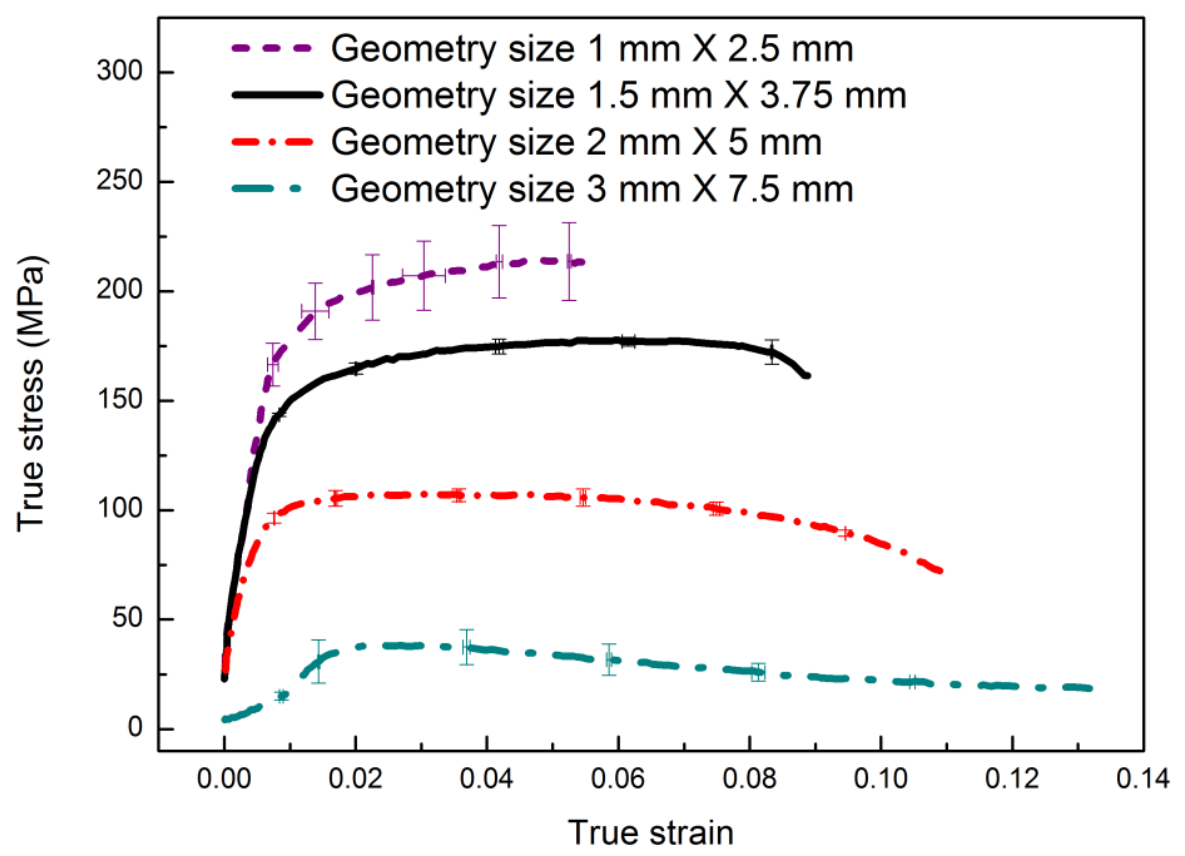

Fig. 7. Uniaxial EA tension behaviors at a current density of $91.1 \mathrm{~A} / \mathrm{mm}^{2}$ for samples with various 
geometry sizes.

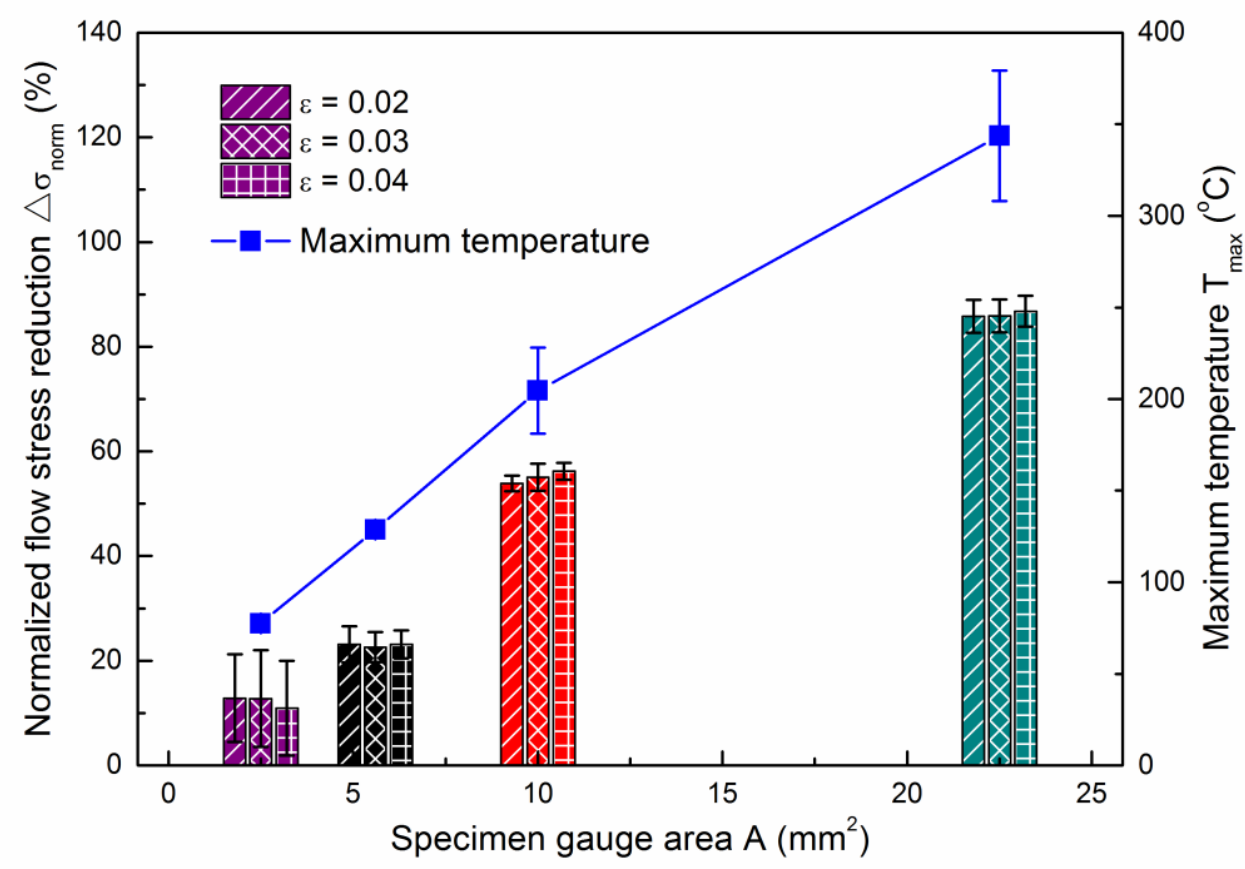

Fig. 8. Normalized flow stress reductions and corresponding peak Joule heating temperatures for specimens having various geometry sizes. 


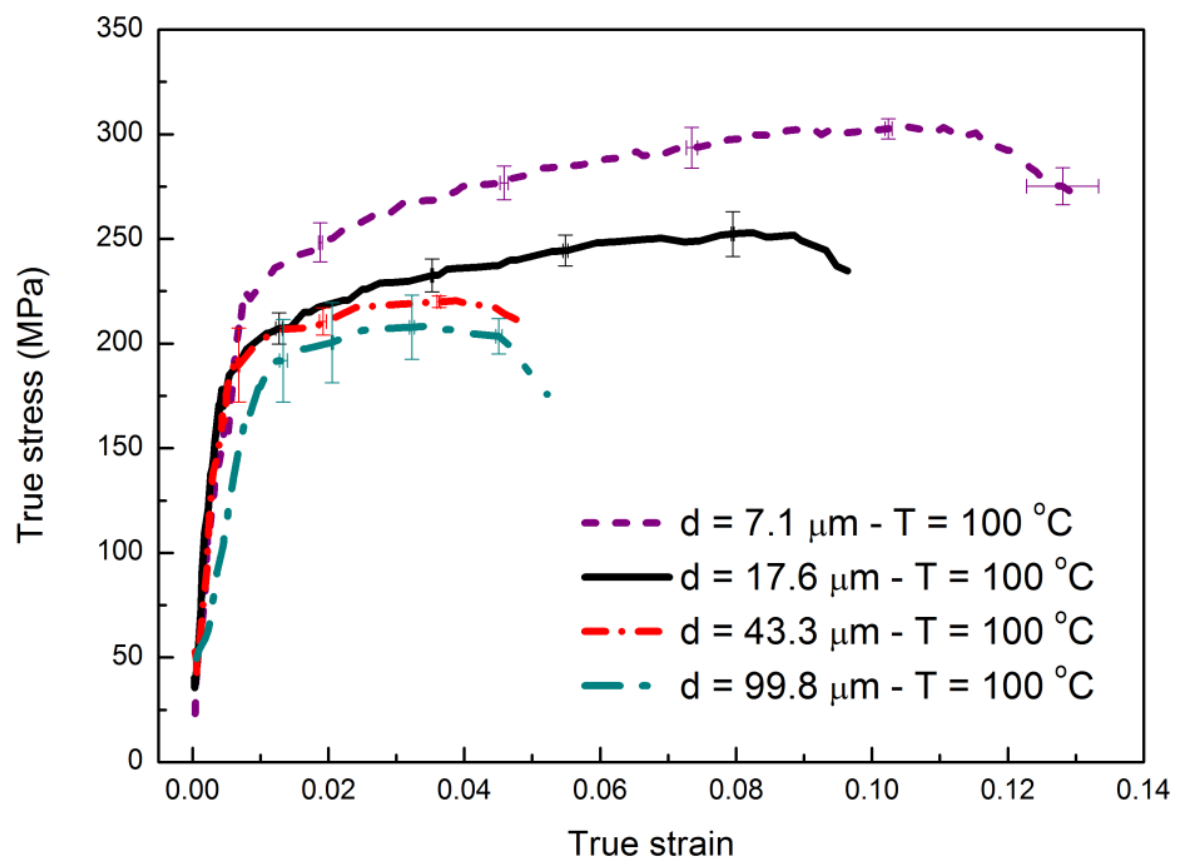

Fig. 9. True stress-strain curves during oven-heated micro-tension of the smallest AZ31 specimens with varying grain sizes. 


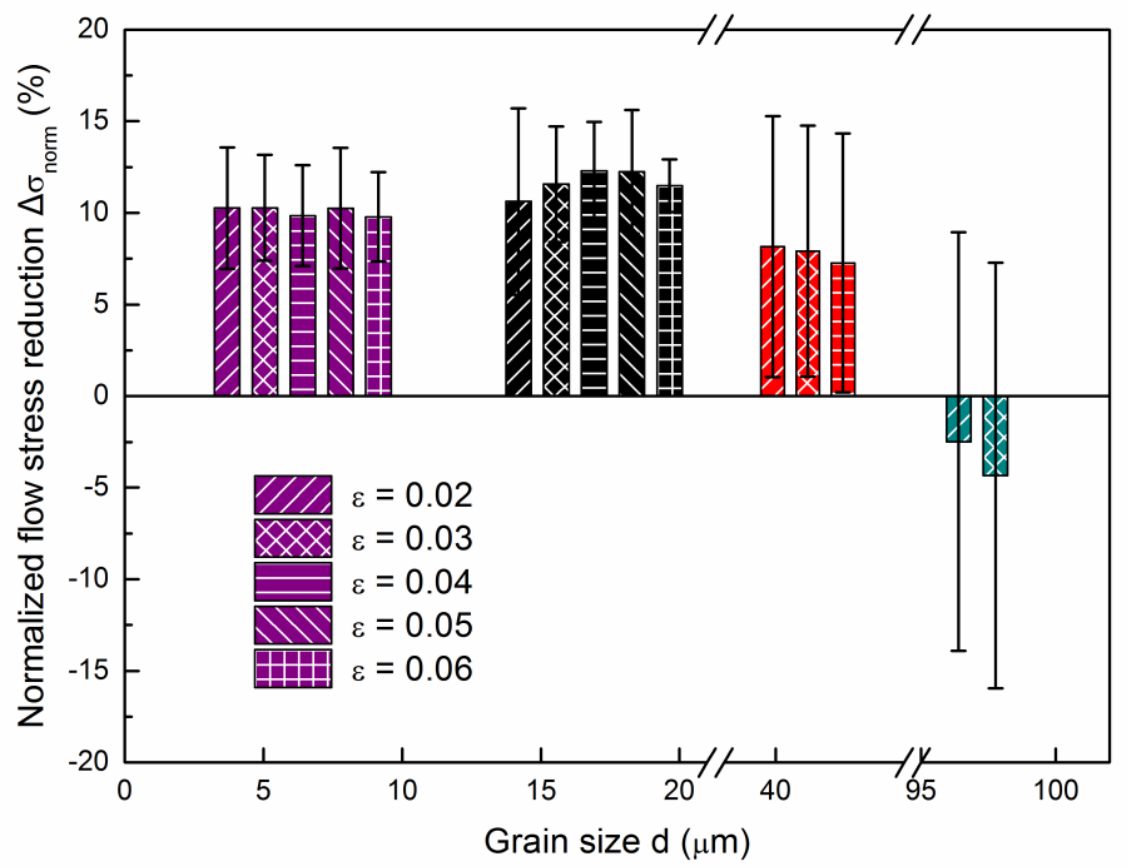

Fig. 10. Normalized flow stress reductions as a function of grain size at different strains for oven-heated micro-tension. 


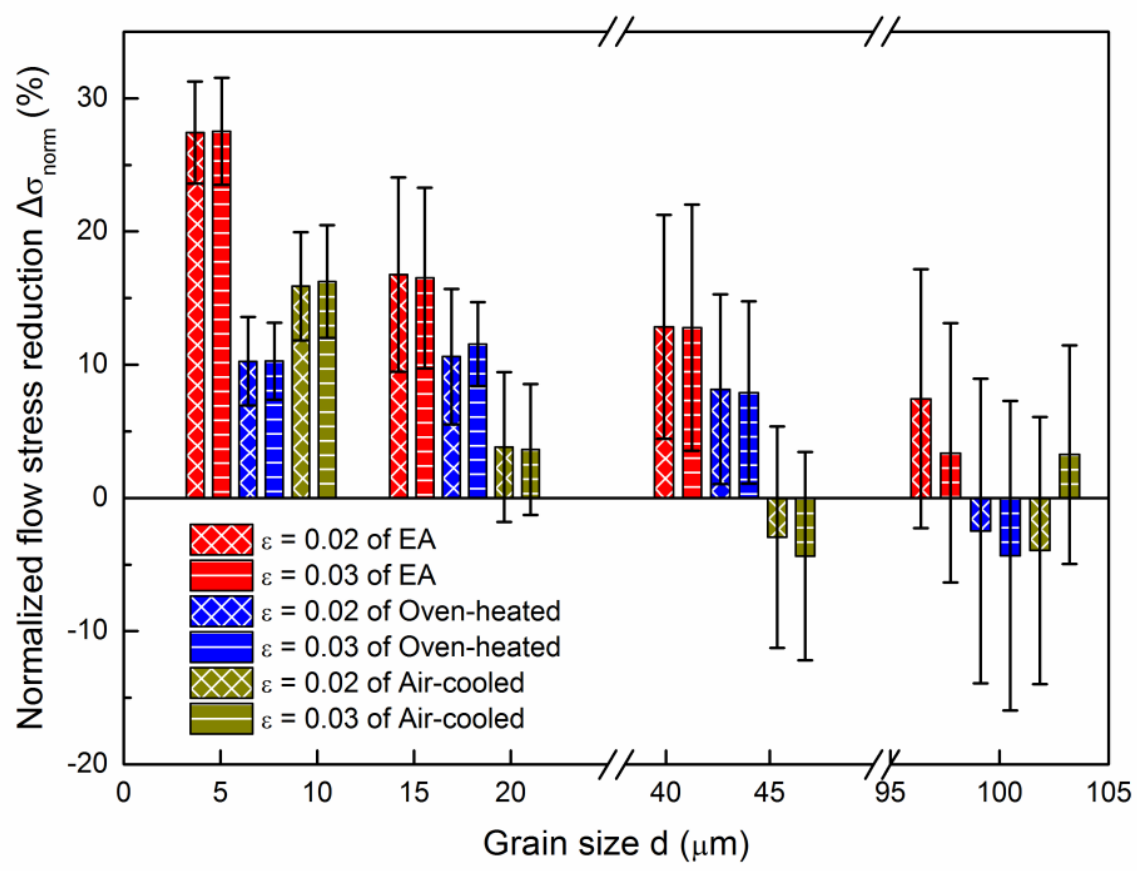

Fig. 11. Comparisons of the normalized flow stress reductions among different test methods at various grain sizes and strains. 


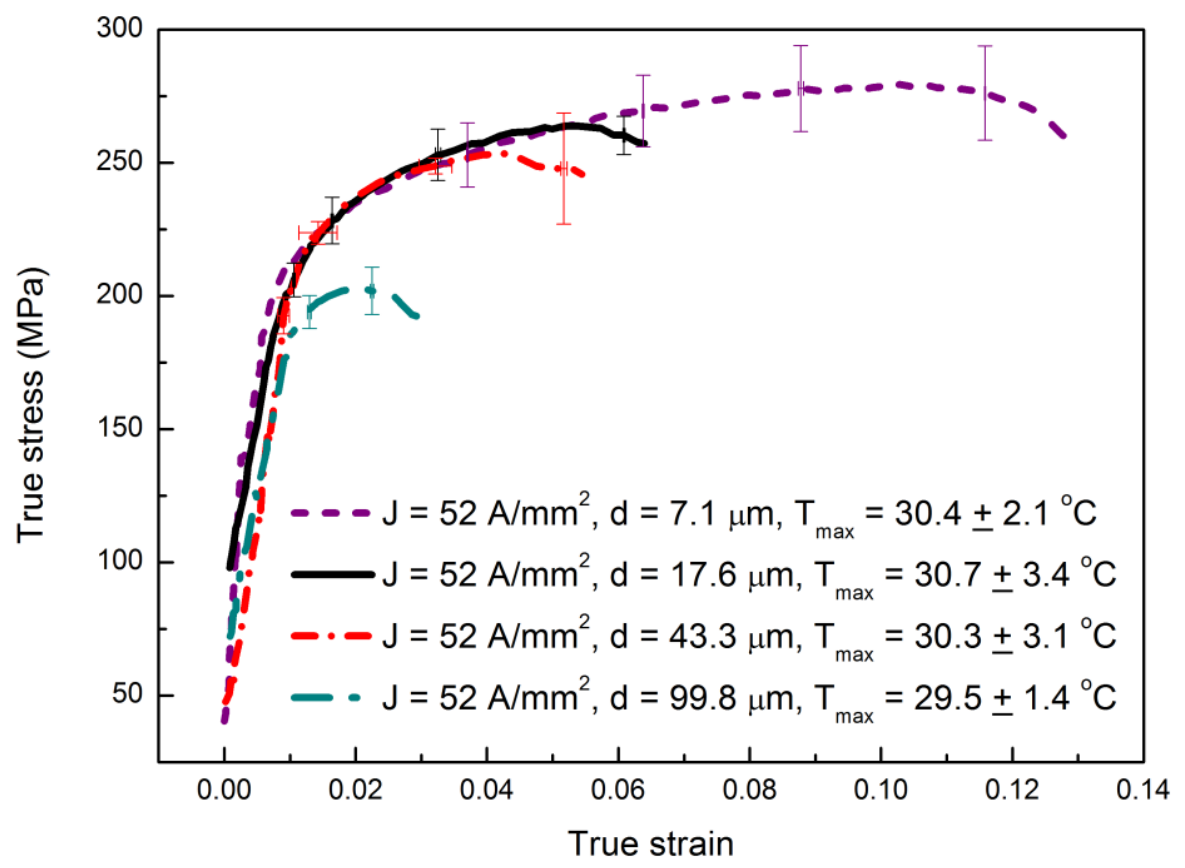

Fig. 12. Uniaxial stress-strain behaviors in air-cooled EA tension tests of samples with various grain sizes. 


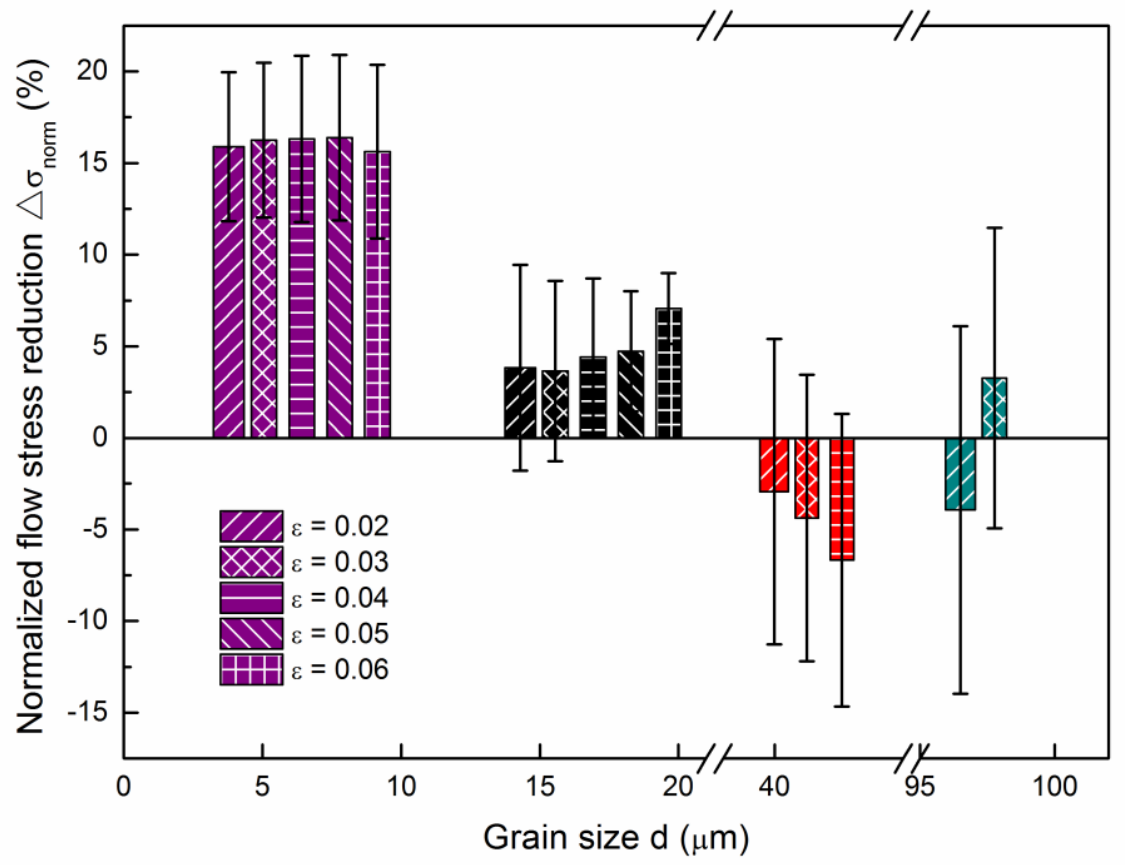

Fig. 13. Normalized flow stress reductions at various strains for different grain sizes in air-cooled

EA micro-tension tests. 


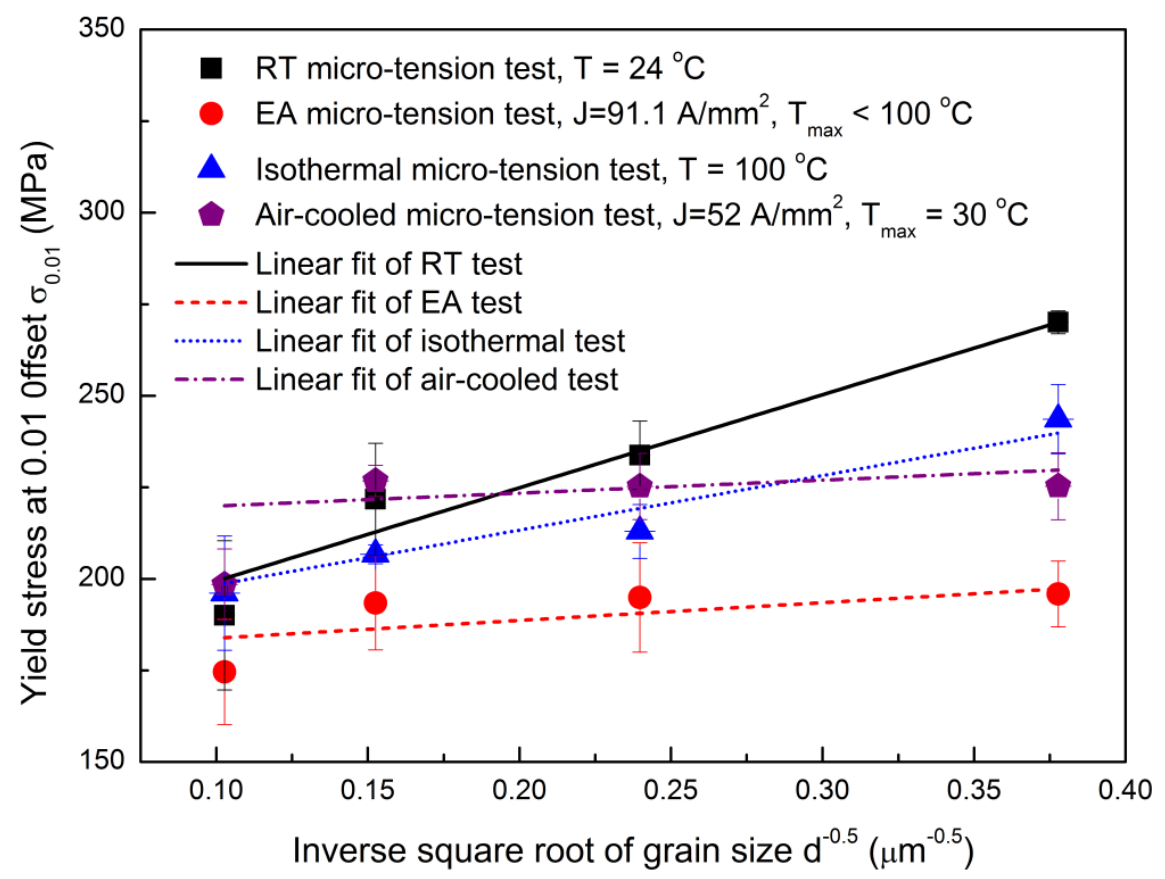

Fig. 14. Comparison of Hall-Petch relationships at offset strain of 0.01 among RT, EA, oven-heated and air-cooled micro-tension tests. 


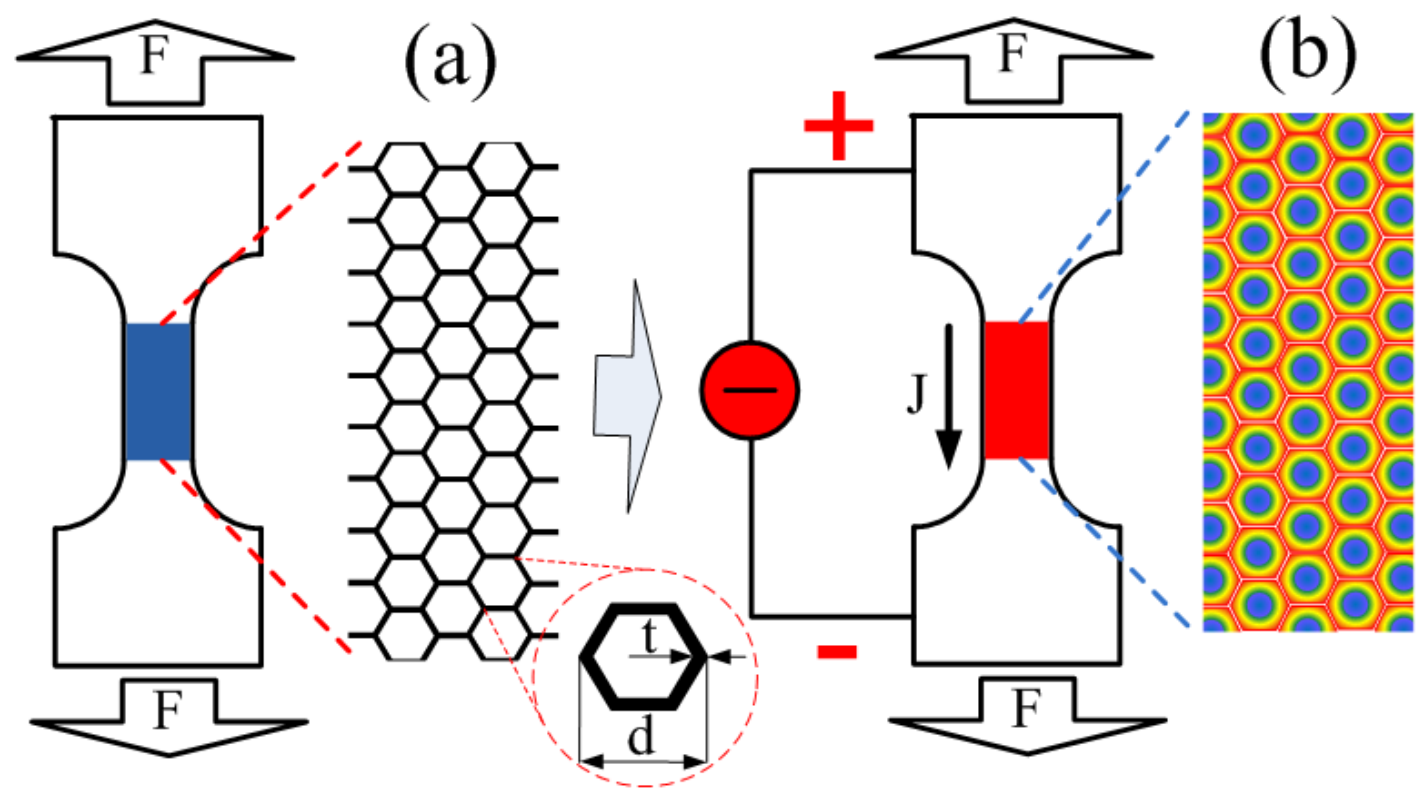

Fig. 15. Schematic diagram of composite material model for Hall - Petch slope in (a) non-EA and (b) EA micro-tension. 